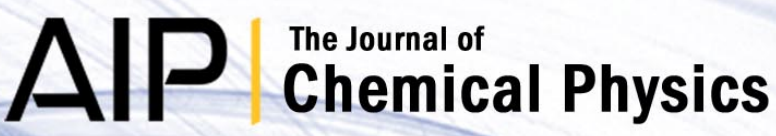

\section{Polarization dependent cavity ring down spectroscopy}

Richard Engeln, Giel Berden, Esther van den Berg, and Gerard Meijer

Citation: J. Chem. Phys. 107, 4458 (1997); doi: 10.1063/1.474808

View online: http://dx.doi.org/10.1063/1.474808

View Table of Contents: http://jcp.aip.org/resource/1/JCPSA6/v107/i12

Published by the American Institute of Physics.

\section{Additional information on J. Chem. Phys.}

Journal Homepage: http://jcp.aip.org/

Journal Information: http://jcp.aip.org/about/about_the_journal

Top downloads: http://jcp.aip.org/features/most_downloaded

Information for Authors: http://jcp.aip.org/authors

\section{ADVERTISEMENT}

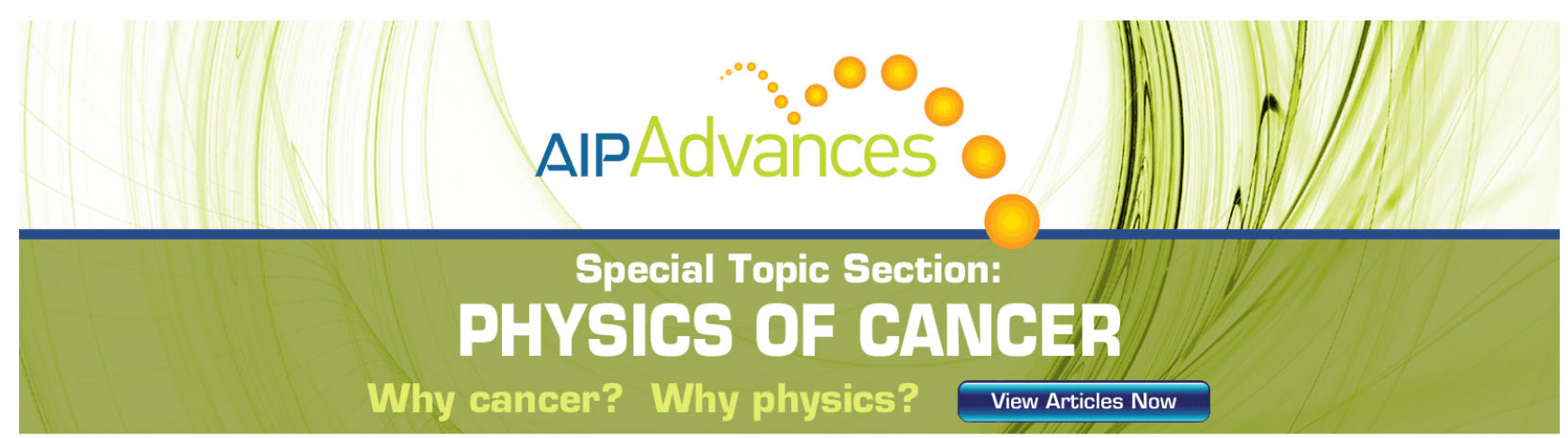




\title{
Polarization dependent cavity ring down spectroscopy
}

\author{
Richard Engeln, ${ }^{\text {a) }}$ Giel Berden, Esther van den Berg, and Gerard Meijer \\ Department of Molecular and Laser Physics, University of Nijmegen, Toernooiveld, 6525 ED Nijmegen, \\ The Netherlands
}

(Received 22 April 1997; accepted 18 June 1997)

\begin{abstract}
We here theoretically outline and experimentally demonstrate that polarization spectroscopy can be combined with cavity ring down (CRD) spectroscopy, thereby retaining the specific advantages of both techniques. The $b^{1} \Sigma_{g}^{+}\left(v^{\prime}=2\right) \leftarrow X^{3} \Sigma_{g}^{-}\left(v^{\prime \prime}=0\right)$ transition of molecular oxygen around $628 \mathrm{~nm}$ is used to demonstrate the possibility to selectively measure either the polarization-dependent absorption or the resonant magneto-optical rotation of gas-phase molecules in the appropriate setup. Just as in CRD absorption spectroscopy, where the rate of absorption is measured, in the here presented polarization-dependent CRD (PDCRD) detection scheme the rate of polarization rotation is measured, which enables the polarization rotation to be quantitatively determined. Apart from studying electro-optic and magneto-optic phenomena on gas-phase species, the PDCRD detection scheme is demonstrated to be applicable to the study of magneto-optical rotation in transparent solid samples as well. (C) 1997 American Institute of Physics. [S0021-9606(97)00836-2]
\end{abstract}

\section{INTRODUCTION}

In 1988 O'Keefe and Deacon demonstrated a new direct absorption spectroscopic technique that can be performed with a pulsed light source and that has a significantly higher sensitivity than obtainable in "conventional" absorption spectroscopy. This so-called cavity ring down (CRD) technique is based upon the measurement of the rate of absorption rather than the magnitude of absorption of a light pulse confined in a closed optical cavity with a high $Q$ factor. ${ }^{1}$ The advantage over normal absorption spectroscopy results from (i) the intrinsic insensitivity of the CRD technique to light source intensity fluctuations, and (ii) the extremely long effective path lengths (many kilometers) that can be realized in stable optical cavities.

Since the technique is based on a pulsed measurement, it can be used in combination with pulsed molecular beams ${ }^{2-4}$ and it can also be used to study dynamical processes via time-resolved absorption measurements. ${ }^{5}$ As long as mirrors with a sufficiently high reflectivity, detectors with a sufficiently fast time response, and tunable (pulsed) light sources are available there is no intrinsic limitation to the spectral region in which CRD can be applied. By now, successful application of CRD spectroscopy has been demonstrated from the uv part of the spectrum ${ }^{6}$ to the ir spectral region. ${ }^{7,8}$ The application of CRD is most straightforward if it can be assumed that the linewidth of the light source can be neglected relative to the width of the molecular absorption. It has been shown, however, that if this assumption is no longer valid, one can still extract the correct absorption coefficient from the measured transients, provided the spectral intensity distribution of the light source is known. ${ }^{6,9}$ As a consequence, it has been experimentally demonstrated that one does not necessarily need a narrow-band pulsed laser to perform a CRD experiment. One might just as well use a polychromatic light source and extract the spectral informa-

${ }^{a)}$ Electronic mail: richarde@ @sci.kun.nl tion after spectrally dispersing the light exiting the cavity, either in a monochromator or by using a Fourier transform spectrometer. ${ }^{8,10}$

The mode structure in the cavity influences the exact temporal profile of the light exiting the cavity. ${ }^{11-13}$ Complications associated with this effect can, however, largely be avoided when an optically stable cavity with a nearcontinuum of modes is used. ${ }^{14}$ High-resolution CRD absorption spectroscopy can be performed when a tunable narrowband continuous laser is coupled into the ring down cavity and the intensity decay in the cavity is determined. The coupling of the narrow-band light into the cavity can be done via locking the laser to the cavity, or, more straightforward, by using the accidental coincidences of the frequency of the modes of the (unstabilized) ring down cavity with the laser frequency. ${ }^{15,16}$

In all the CRD experiments reported to date polarized laser radiation has been used for absorption measurements. By putting a polarization selective optical element in front of the detector, it should in principle be possible to measure the optical rotation of the plane of polarization of the incoming beam upon passage through the ring down cavity. With a polarization analyzer that selects radiation polarized parallel to that of the incoming beam placed in front of the light detector, in addition to the "normal" absorption, rotation of the plane of polarization in the ring down cavity will be detected as an apparent absorption, i.e., as a shortening of the ring down time. With the analyzer rotated (almost) $90^{\circ}$ relative to the plane of polarization of the incoming beam, there will still be shortening of the cavity decay time due to absorption but optical rotation will cause a time-dependent increase in the detected signal, i.e., it will appear as an apparent lengthening of the ring down time ("cavity ring up"). When both signals are measured simultaneously, one can discriminate between the effects of absorption and optical rotation.

Magnetic rotation spectroscopy ${ }^{17}$ based on the Zeeman effect, is a zero-background absorption technique that is con- 
siderably more sensitive than direct single- or double-beam absorption. This technique has been applied to a variety of paramagnetic species, and the resonant magneto-optic spectrum of the $v^{\prime}=0 \leftarrow v^{\prime \prime}=0$ transition of the atmospheric band of molecular oxygen has recently been reported. ${ }^{18}$ The sensitivity of the technique can in principle be increased by multipassing through the sample (the polarization rotates further with each traversal). In practice, however, multipassing is often found to be limited by the deterioration of the degree of polarization with each mirror reflection. ${ }^{19,20}$

In this paper we report the first experimental demonstration of the combination of polarization spectroscopy with cavity ring down spectroscopy. The $b^{1} \Sigma_{g}^{+}\left(v^{\prime}=2\right)$ $\leftarrow X^{3} \Sigma_{g}^{-}\left(v^{\prime \prime}=0\right)$ magnetic dipole transition of molecular oxygen around $628 \mathrm{~nm}$ is used to demonstrate the possibility to selectively measure either the polarization dependent absorption or the resonant magneto-optical rotation. The polarization-dependent CRD (PDCRD) detection scheme outlined here offers a significant improvement in sensitivity and utility over the individual methods. In contrast to most other schemes for polarization spectroscopy, the PDCRD scheme is not completely background-free, and is therefore not overly sensitive to the above-mentioned deterioration of the degree of polarization upon multipassing through the cavity. A theoretical analysis of the expected ring down transients in PDCRD spectroscopy is given. In the Voigt configuration (external magnetic field perpendicular to the axis of the cavity) the detection limit for molecular oxygen is lowered by more than an order of magnitude in the PDCRD setup relative to the "conventional" CRD setup. In addition, there is a sign-difference for absorptions polarized parallel and perpendicular to the magnetic field, which aids considerably in the assignment. In the Faraday configuration (external magnetic field parallel to the axis of the cavity) the experimental arrangement can be made such that one is exclusively sensitive to optical rotation, and no effect of (polarization dependent) absorption is observed. In the PDCRD detection scheme, the rate of polarization rotation is measured, which enables the rotation to be quantitatively determined. In the experiments presented here a noise-equivalent polarization rotation of $10^{-8} \mathrm{rad} / \mathrm{cm}$ is demonstrated. Apart from studying electro-optic and magneto-optic phenomena on gas-phase species, the PDCRD technique is shown to be applicable to the study of optical rotation of transparent solid samples as well.

\section{THEORY}

In a typical CRD experiment a short light pulse is coupled into a stable optical cavity, formed by two highly reflecting plano-concave mirrors. The fraction of light entering the cavity on one side "rings" back and forth many times between the two mirrors. The time behavior of the light intensity inside the cavity $I_{\mathrm{CRD}}(t)$, can be monitored by the small fraction of light that is transmitted through the other mirror. If the only loss factor in the cavity is the reflectivity loss of the mirrors, one can show that the light intensity inside the cavity decays exponentially in time with a decay constant $\tau$, the "ring down time," given by

$$
\tau=\frac{d}{c(|\ln R|)} .
$$

Here $d$ is the optical path length between the mirrors, $c$ is the speed of light, and $R$ is the reflectivity of the mirrors. If $R$ is close to unity, the approximation $\tau=d / c(1-R)$ can be made.

If there is additional loss inside the cavity due to the presence of absorbing and light scattering species, the light intensity inside the cavity will still decay exponentially in time provided the absorption follows Beer's law. It can be shown that in this case the ring down transient is proportional to:

$$
I_{\mathrm{CRD}}(t) \propto \int_{0}^{\infty} I(\nu) e^{-t / \tau(\nu)} d \nu
$$

where $I(\nu)$ is the spectral distribution of the light that makes it into the cavity and where $\tau(\nu)$ is given by

$$
\tau(\nu)=\frac{d}{c\left(|\ln R(\nu)|+\sum_{i} \sigma_{i}(\nu) \int_{0}^{d} N_{i}(x) d x\right)}
$$

and the sum is over all light scattering and absorbing species with frequency-dependent cross sections $\sigma_{i}(\nu)$ and a lineintegrated number density $\int_{0}^{d} N_{i}(x) d x$. The product of the frequency-dependent absorption cross section with the number density $N_{i}(x)$ is commonly expressed as the absorption coefficient $\kappa_{i}(\nu, x)$. In an experiment it is most convenient to record $1 / c \tau(\nu)$ as a function of frequency, i.e., to record the total cavity loss as a function of frequency, as this is directly proportional to the absorption coefficient, apart from an offset which is mainly determined by the finite reflectivity of the mirrors.

When a linearly polarized laser beam traverses the medium in the cavity, the intensity of the light can decay due to absorption and, if a magnetic field is present, the plane of polarization can rotate due to dispersion, i.e., due to different velocities for different polarization directions (magnetic circular birefringence). If the absorptions are polarization dependent, these will also lead to an effective rotation of the plane of polarization (magnetic dichroism).

In the description given below, we assume that the reflectivity of the mirrors is independent of polarization and that the mirrors do not cause any optical rotation by themselves. In addition, we assume that an ideal polarizer is used, both to define the plane of polarization of the incoming beam and to analyze the polarization of the light exiting the cavity. It is also assumed that polarization-dependent absorption and optical rotation is homogeneous over a length $l \leqslant d$. The extension to more realistic situations is straightforward and in presenting the experimental results, we will briefly discuss the (minor) influence of the actual deviations from this ideal situation. 


\section{A. The Voigt configuration}

In the Voigt configuration a homogeneous magnetic field B is applied perpendicular to the axis of the cavity over a length $l$. The plane of polarization of the incoming laser beam makes an angle $\phi_{B}$ with the direction of the magnetic field. In the magnetic field we distinguish between absorptions induced by light that is either polarized parallel or perpendicular to the magnetic field, having absorption coefficients $\kappa_{\|}(\nu)$ and $\kappa_{\perp}(\nu)$, respectively. Similarly, a distinction is made between the refractive index for light that is polarized either parallel $\left[n_{\|}(\nu)\right]$ or perpendicular $\left[n_{\perp}(\nu)\right]$ to the magnetic field. If the total integrated light intensity exiting the cavity is measured as a function of time, i.e., when CRD detection without polarization selection in the detection step is performed, the expression for $1 / c \tau(\nu)$ is given by

$$
\frac{1}{c \tau(\nu)}=\frac{1}{c \tau_{0}}+\frac{l}{d}\left[\kappa_{\|}(\nu) \cos ^{2} \phi_{B}+\kappa_{\perp}(\nu) \sin ^{2} \phi_{B}\right],
$$

where $1 / c \tau_{0}$ represents the remaining, polarization independent, cavity losses. As expected, any polarization rotation that might occur in the magnetic field will not be noticed in this case.

When of all the light exiting the cavity only the polarization component that makes an angle $\phi_{D}$ with the incoming polarization is detected, the time dependence of this light intensity will be given by (see the Appendix)

$$
\begin{aligned}
& I_{\phi_{D}}(\nu, t)=I_{0} e^{-t / \tau_{0}}\left[\cos ^{2}\left(\phi_{D}\right)-\frac{c l t \cos \left(\phi_{D}\right)}{d}\right. \\
& \times\left[\cos \left(\phi_{B}\right) \cos \left(\phi_{D}-\phi_{B}\right) \kappa_{\|}(\nu)\right. \\
& \left.-\sin \left(\phi_{B}\right) \sin \left(\phi_{D}-\phi_{B}\right) \kappa_{\perp}(\nu)\right] \\
& +\frac{1}{2} \sin \left(2 \phi_{B}\right) \sin \left(2\left(\phi_{D}-\phi_{B}\right)\right) \\
& \left.\times\left[1-\cos \left(\frac{\omega l t\left(n_{\|}(\nu)-n_{\perp}(\nu)\right)}{d}\right)\right]\right],
\end{aligned}
$$

where it is assumed that a total light intensity $I_{0}$ is transmitted through the end mirror at time $t=0$, and where it is assumed that both the polarization-dependent absorption and the optical rotation is small, such that the term involving their product can be neglected. In the above formula $\omega$ is the angular frequency of the light. When the analyzer and the polarizer are exactly crossed, only the last term remains in the above formula yielding a ring down transient that contains the product of an exponentially decaying part and a part that, for small overall rotations, rises quadratically with time. In practice, however, there will always be a non-negligible fraction of polarization distortion due to mirror imperfections, etc., and it is more realistic to look at angles $\phi_{D}$ close to, but not identical to, $90^{\circ}$. For this situation, the terms containing the polarization-dependent absorption will dominate and the dispersion term can in first order be neglected. For weak absorptions, the ring down transient is observed to be again an exponentially decaying function of time, with:

$$
\begin{aligned}
\frac{1}{c \tau_{\phi_{D}}(\nu)}= & \frac{1}{c \tau_{0}}+\frac{l}{d \cos \left(\phi_{D}\right)}\left[\cos \left(\phi_{B}\right) \cos \left(\phi_{D}-\phi_{B}\right)\right. \\
& \left.\times \kappa_{\|}(\nu)-\sin \left(\phi_{B}\right) \sin \left(\phi_{D}-\phi_{B}\right) \kappa_{\perp}(\nu)\right] .
\end{aligned}
$$

If the time dependence of the light intensity along two mutually orthogonal polarization directions is measured simultaneously, the difference between the cavity losses measured for either direction is given by

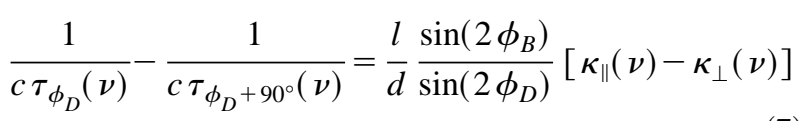

and this quantity can be accurately experimentally determined. Compared to "conventional" CRD absorption spectroscopy there is a gain in intensity by a factor $\sin \left(2 \phi_{B}\right) / \sin \left(2 \phi_{D}\right)$, and there is a sign-difference between absorptions polarized parallel or perpendicular to the external magnetic field. In addition, most of the "noise" in the spectrum of $1 / c \tau(\nu)$ versus frequency, noise that is probably associated with variations of the mode structure in the cavity, is experimentally found to be identical in either polarization direction, and therefore cancels out in the difference signal.

\section{B. The Faraday configuration}

In the Faraday configuration a homogeneous magnetic field $\mathbf{B}$ is applied parallel to the axis of the cavity over a length $l$. The linearly polarized incoming laser beam is now to be viewed as the sum of equal amounts of right- and left-circularly polarized light. In the magnetic field we distinguish between absorptions induced by either one of the circularly polarized components, having absorption coefficients $\kappa_{+}(\nu)$ and $\kappa_{-}(\nu)$, respectively. Similarly, a distinction is made between the refractive indices $n_{+}(\nu)$ and $n_{-}(\nu)$ for right- and left-circularly polarized light. If the total integrated light intensity exiting the cavity is measured as a function of time, the expression for $1 / c \tau(\nu)$ is given by

$$
\frac{1}{c \tau(\nu)}=\frac{1}{c \tau_{0}}+\frac{l}{2 d}\left[\kappa_{+}(\nu)+\kappa_{-}(\nu)\right] .
$$

Also in this configuration, any polarization rotation will not be noticed when there is no polarization selection in the detection step.

When of all the light exiting the cavity only the polarization component that makes an angle $\phi_{D}$ with the incoming polarization is detected, the time dependence of this light intensity will be given by (see the Appendix)

$$
\begin{aligned}
I_{\phi_{D}}(\nu, t)= & \frac{1}{4} I_{0} e^{-t / \tau_{0}}\left[e^{-\kappa_{+}(\nu) c l t / d}+e^{-\kappa_{-}(\nu) c l t / d}\right. \\
& +2 e^{-\left(\kappa_{+}(\nu)+\kappa_{-}(\nu)\right) c l t / 2 d} \cos \left(2 \phi_{D}\right. \\
& \left.\left.+\frac{\omega l t\left(n_{+}(\nu)-n_{-}(\nu)\right)}{d}\right)\right] .
\end{aligned}
$$



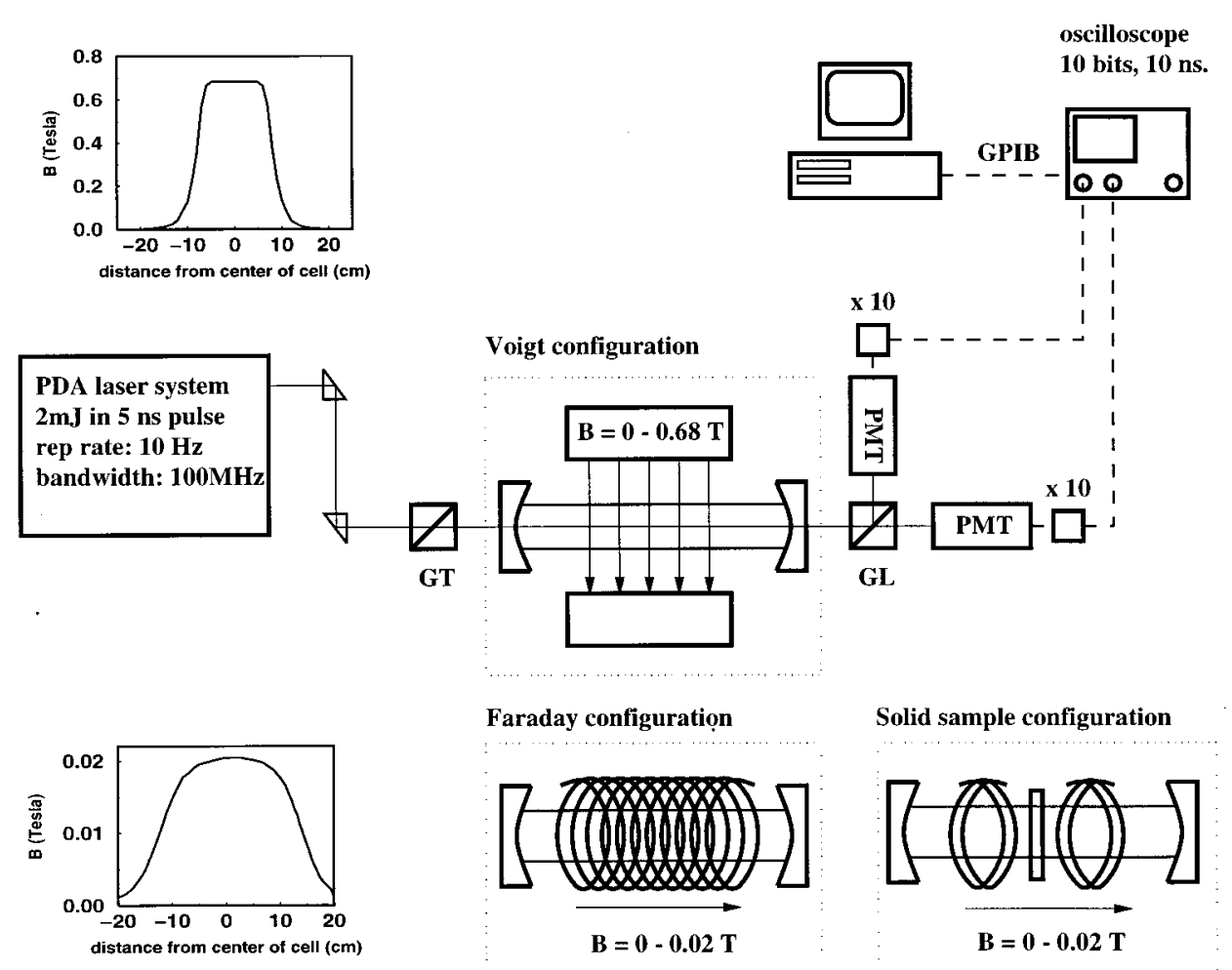

Faraday configuration

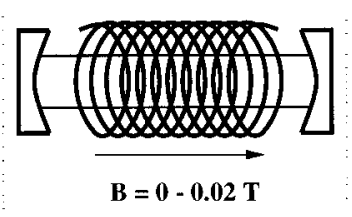

Solid sample configuration

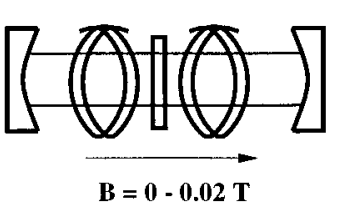

FIG. 1. Schematic view of the experimental setup. Pulsed narrow-band laser radiation is coupled into an optically stable ring down cavity. Prior to entering the cavity, the light passes through a Glan-Thompson polarizer (GT). The light exiting the cavity is split by a Glan-Laser polarizer (GL) into two mutually orthogonally polarized components that are recorded independently. The measured magnetic field strength along the axis of the cavity is indicated in the inset both for the Voigt and for the Faraday configuration. In the Faraday configuration for the solid sample, two Helmholtz coils are used to create the magnetic field.

In the limit of weak absorptions, and for small values of the overall polarization rotation this expression again represents a single-exponentially decaying transient with

$$
\begin{aligned}
\frac{1}{c \tau_{\phi_{D}}(\nu)}= & \frac{1}{c \tau_{0}}+\frac{l\left(\kappa_{+}(\nu)+\kappa_{-}(\nu)\right)}{2 d} \\
& +\frac{\omega l\left(n_{+}(\nu)-n_{-}(\nu)\right)}{c d} \tan \left(\phi_{D}\right) .
\end{aligned}
$$

This implies that there is always a contribution from the polarization-dependent absorption in addition to a contribution due to optical rotation. By making the angle $\phi_{D}$ close to $90^{\circ}$, the effect due to optical rotation is enhanced relative to the effect of polarization dependent absorption. If the time dependence of the light intensity along two mutually orthogonal polarization directions is measured simultaneously, the difference between the values for $1 / c \tau(\nu)$ found for the two directions is solely determined by the dispersion term, and is given by

$$
\frac{1}{c \tau_{\phi_{D}}(\nu)}-\frac{1}{c \tau_{\phi_{D}+90^{\circ}}(\nu)}=\frac{2 \omega l}{c d \sin \left(2 \phi_{D}\right)}\left[n_{+}(\nu)-n_{-}(\nu)\right] \text {. }
$$

In this setup one is therefore selectively sensitive to polarization rotation, a quantity that cannot be measured in "conventional" CRD spectroscopy. As in CRD absorption spectroscopy, where the rate of absorption is measured, in
PDCRD the rate of optical rotation is measured, and this enables the optical rotation to be put on an absolute scale.

\section{EXPERIMENTAL SETUP}

A schematic representation of the experimental setup is depicted in Fig. 1. Pulsed narrow-band laser radiation is obtained from a pulsed amplified single-mode $\mathrm{cw}$ ring dye laser system. The laser system runs at $10 \mathrm{~Hz}$ and delivers $5 \mathrm{~ns}$ duration pulses with approximately $1-10 \mathrm{~mJ}$ of energy. The full width at half maximum of the spectral profile of the laser pulse is about $100 \mathrm{MHz}$, close to the Fourier transform limit. Continuous, mode-hop free scans, can be made over $1.5 \mathrm{~cm}^{-1}$ in the visible region of the spectrum. The absolute frequency of the cw laser is determined via simultaneous measurement of the absorption spectrum of molecular iodine in a room-temperature cell. To be able to linearize the spectral scans, the transmission spectrum of a Fabry-Perot étalon with a free spectral range of $299 \mathrm{MHz}$ is recorded simultaneously.

The ring down cavity is formed by two plano-concave mirrors with a diameter of $25 \mathrm{~mm}$ and a radius of curvature of $-1 \mathrm{~m}$, placed at a distance slightly less than $50 \mathrm{~cm}$ apart. The mirrors are coated for an optimum reflectivity at 620 $\mathrm{nm}$, with a quoted reflectivity of better than 0.9999 (Newport, SuperMirrors). The mirrors act at the same time as windows for the closed-off pyrex cell, which is filled with pure 
molecular oxygen (5-420 mbar) for the experiments reported here. In selecting the mirrors it is crucial to have coatings that do not deteriorate the degree of polarization of the light inside the cavity to a large extent. Many of the multilayer reflection coatings are actually optically active and will slightly rotate the plane of polarization on every reflection. The degree to which this occurs depends on details of the production process for the mirrors. Before entering the ring down cavity the incoming laser beam passes through a Glan-Thompson polarizer with an extinction of $10^{-5}$. In the Voigt configuration, an electromagnet (Spectromagnetic Industries) capable of producing magnetic fields up to $0.68 \mathrm{~T}$ is used and the polarization of the incoming beam relative to the direction of the magnetic field, i.e., the angle $\phi_{B}$, can be set with an accuracy of about $1^{\circ}$. The magnetic field is homogeneous over a length of $12 \mathrm{~cm}$, and drops on either side to zero field over a distance of several $\mathrm{cm}$. In the Faraday configuration an electromagnet with a $10 \mathrm{~cm}$ diameter bore and a length of $27 \mathrm{~cm}$ is used. With this magnet, only fields up to $0.02 \mathrm{~T}$ could be produced. In the inset of Fig. 1 the experimentally determined magnitude of the magnetic field along the axis of the ring down cavity is shown for both the Voigt and the Faraday configuration.

The light exiting the ring down cavity is passed through a Glan-Laser polarizer, and split into its two mutually orthogonally polarized components (extinction of $10^{-5}$ ). The axis of this Glan-Laser analyzer can be oriented relative to the polarization direction of the incoming beam with an accuracy of $0.1^{\circ}$. In order to ensure that all transverse modes are detected with equal probability the two photomultiplier tubes (PMT) that are used to measure the ring down transients are placed close to the analyzer. The signals of both PMTs are amplified and displayed on a fast (10 ns) and highresolution (10 bit) digital oscilloscope (LeCroy 9430). At every wavelength position some 50 transients, every transient recorded over a $20 \mu$ s duration time interval, are summed on the 16-bit on-board memory of the oscilloscope and read out by a PC via a GPIB interface connection. The characteristic ring down time is determined by fitting the natural logarithm of the data to a straight line, using a weighted least-squares fitting algorithm. The time constant that describes the decay of the empty cavity in our setup is around $9 \mu \mathrm{s}$, corresponding to an effective reflection coefficient of $R \approx 0.9998$ in the spectral region of the $2 \leftarrow 0$ transition of the atmospheric band of molecular oxygen around $628 \mathrm{~nm}$.

\section{EXPERIMENTAL RESULTS AND DISCUSSION}

For demonstration purposes, all the spectra that we will show are those of the ${ }^{P} P_{1}(1)$ line of the $b^{1} \Sigma_{g}^{+}\left(v^{\prime}=2\right)$ $\leftarrow X^{3} \Sigma_{g}^{-}\left(v^{\prime \prime}=0\right)$ magnetic dipole transition in ${ }^{16} \mathrm{O}_{2}$ at $15899.55 \mathrm{~cm}^{-1}$. ${ }^{22}$ The ${ }^{P} P_{1}(1)$ line originates from either one of the three possible $M$ components of the $J=1$ level in the electronic ground state and ends up in the rotationless level of the electronically excited state. In Fig. 2 the calculated Zeeman splitting of the $J=1, N=1$ level in the electronic ground state is shown as a function of the magnetic-

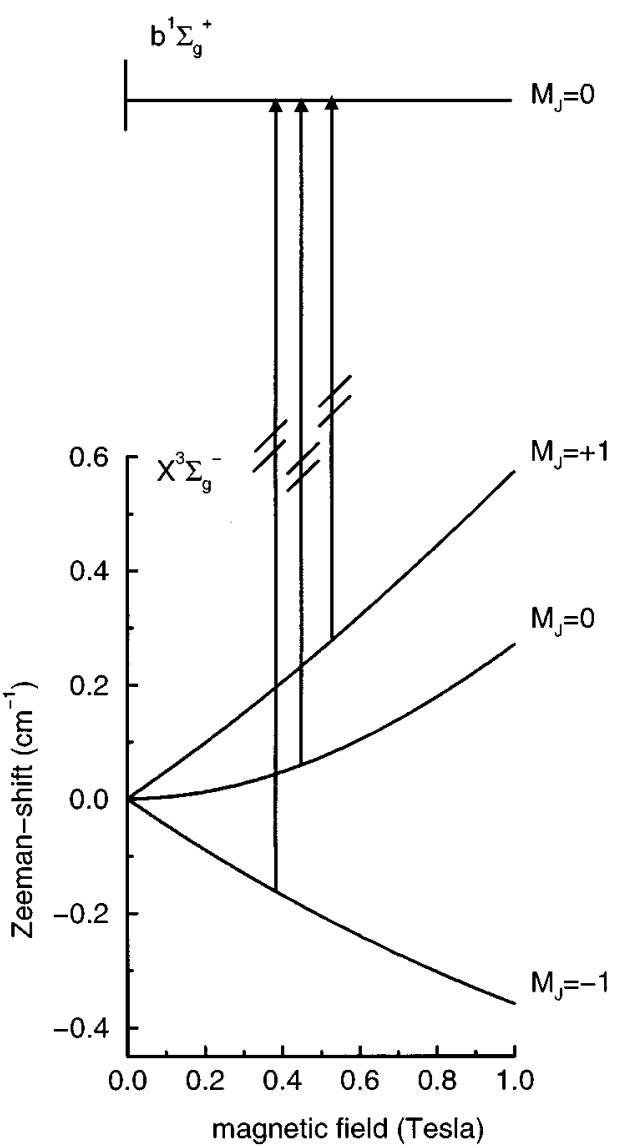

FIG. 2. Calculated Zeeman shift as a function of the magnetic-field strength for the $J=1, N=1$ rotational level in the $X^{3} \Sigma_{g}^{-}(v=0)$ ground state of ${ }^{16} \mathrm{O}_{2}$. The three $\Delta M$ components of the ${ }^{P} P_{1}(1)$ line of the $b^{1} \Sigma_{g}^{+}\left(v^{\prime}\right.$ $=2) \leftarrow X^{3} \Sigma_{g}^{-}\left(v^{\prime \prime}=0\right)$ band, located at $15899.55 \mathrm{~cm}^{-1}$ under field-free conditions, are schematically indicated.

field strength. ${ }^{23}$ It is observed that in fields larger than $0.2 \mathrm{~T}$ there is a significant contribution from the second-order Zeeman effect, resulting in a measurable shift of all the three $\Delta M$ transitions from their field-free position. It is assumed that the Zeeman shift in the electronically excited state can be neglected. To explicitly demonstrate the species selectivity of the PDCRD technique, a small amount of water vapor (several mbar) is leaked into the ring down cavity as well, and the $4_{14} \leftarrow 3_{13}$ rotational line of the $(1,1,3) \leftarrow(0,0,0)$ vibrational band of $\mathrm{H}_{2} \mathrm{O}$ at $15899.045 \mathrm{~cm}^{-1}$ is recorded in the same spectral scan. ${ }^{24}$

\section{A. The Voigt configuration}

In Fig. 3 the CRD absorption spectrum of the ${ }^{P} P_{1}(1)$ transition as measured with 420 mbar of $\mathrm{O}_{2}$ in the cell is shown in the Voigt configuration, without polarization selection in the detection step. In the upper part, measured in zero magnetic field, a single line is found with a full width at half maximum (FWHM) of $0.068 \mathrm{~cm}^{-1}$, determined by the combined effect of Doppler broadening (Gaussian line with FWHM of $0.035 \mathrm{~cm}^{-1}$ ) and pressure broadening (Lorentzian with FWHM of $\left.0.050 \mathrm{~cm}^{-1}\right) .{ }^{25}$ With the magnetic field turned up to $0.68 \mathrm{~T}$, CRD absorption spectra as measured for 


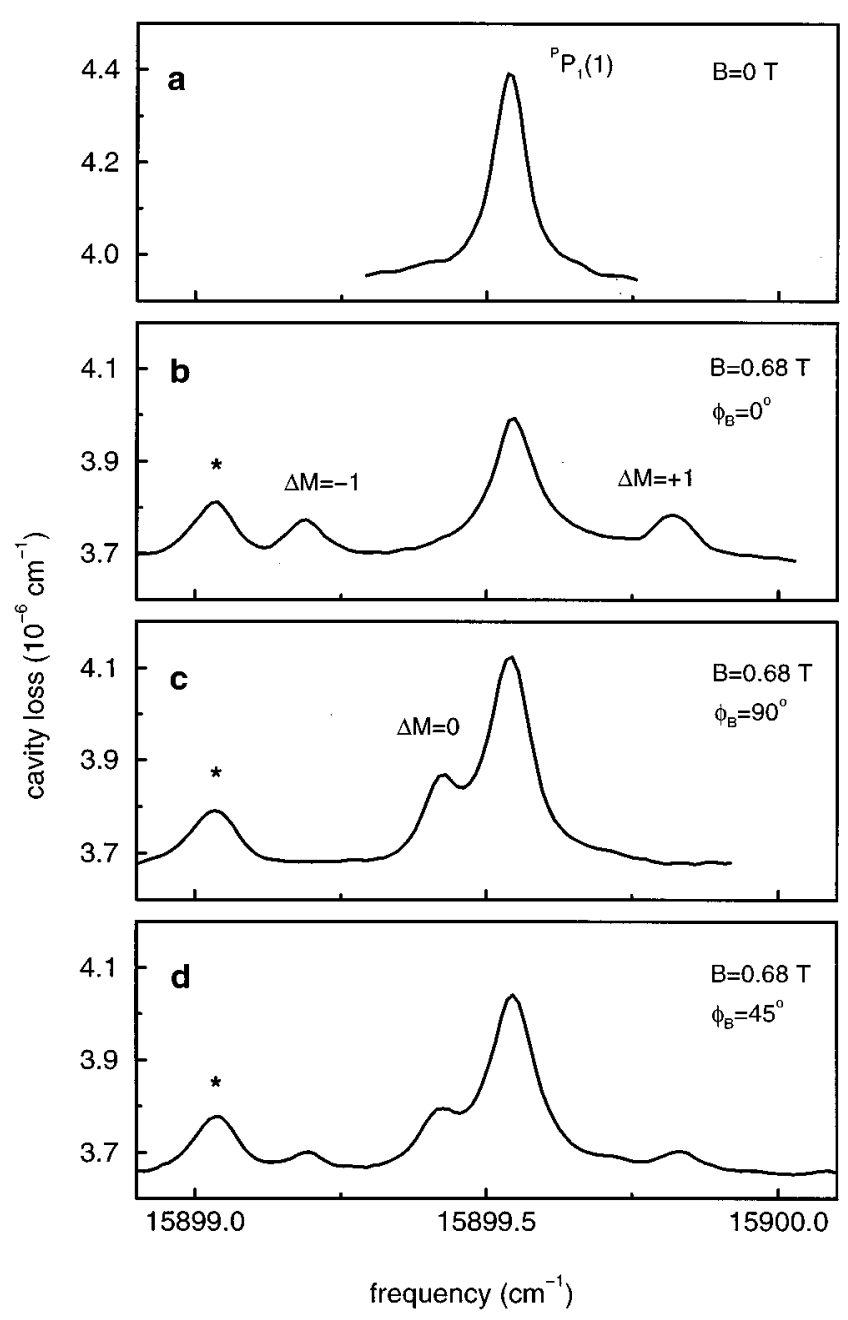

FIG. 3. The CRD absorption spectrum of the ${ }^{P} P_{1}(1)$ line of the $b^{1} \Sigma_{g}^{+}\left(v^{\prime}\right.$ $=2) \leftarrow X^{3} \Sigma_{g}^{-}\left(v^{\prime \prime}=0\right)$ magnetic dipole transition in ${ }^{16} \mathrm{O}_{2}$, measured at a pressure of $420 \mathrm{mbar}$, without polarization selection in the detection step. In (a) no magnetic field is applied. In (b)-(d) a magnetic field of $0.68 \mathrm{~T}$ is applied in the Voigt configuration and the plane of polarization of the incoming light makes an angle $\phi_{B}=0^{\circ}, \phi_{B}=90^{\circ}$, and $\phi_{B}=45^{\circ}$ with the $\mathbf{B}$ field, respectively. The line indicated with an asterisk is due to water absorption.

three different angles of the plane of polarization relative to the direction of the magnetic field are shown, under otherwise identical conditions. With the laser polarization parallel to the magnetic field, i.e., $\phi_{B}=0^{\circ}$, only the $\Delta M= \pm 1$ transitions are seen to absorb, in agreement with the selection rules for magnetic dipole transitions. With the laser polarization perpendicular to the magnetic field, i.e., $\phi_{B}=90^{\circ}$, only the $\Delta M=0$ transition, which is about twice as strong as the $M$-changing transitions, is observed. In agreement with the expression given in Eq. (4), all $\Delta M$ transitions are observed when $\phi_{B}$ is set equal to $45^{\circ}$, albeit with a factor of 2 lower intensity than in either of the two situations just mentioned. As the magnetic field does not cover the whole absorption cell, there is always a remaining absorption at the field-free position. In addition, the water absorption line around
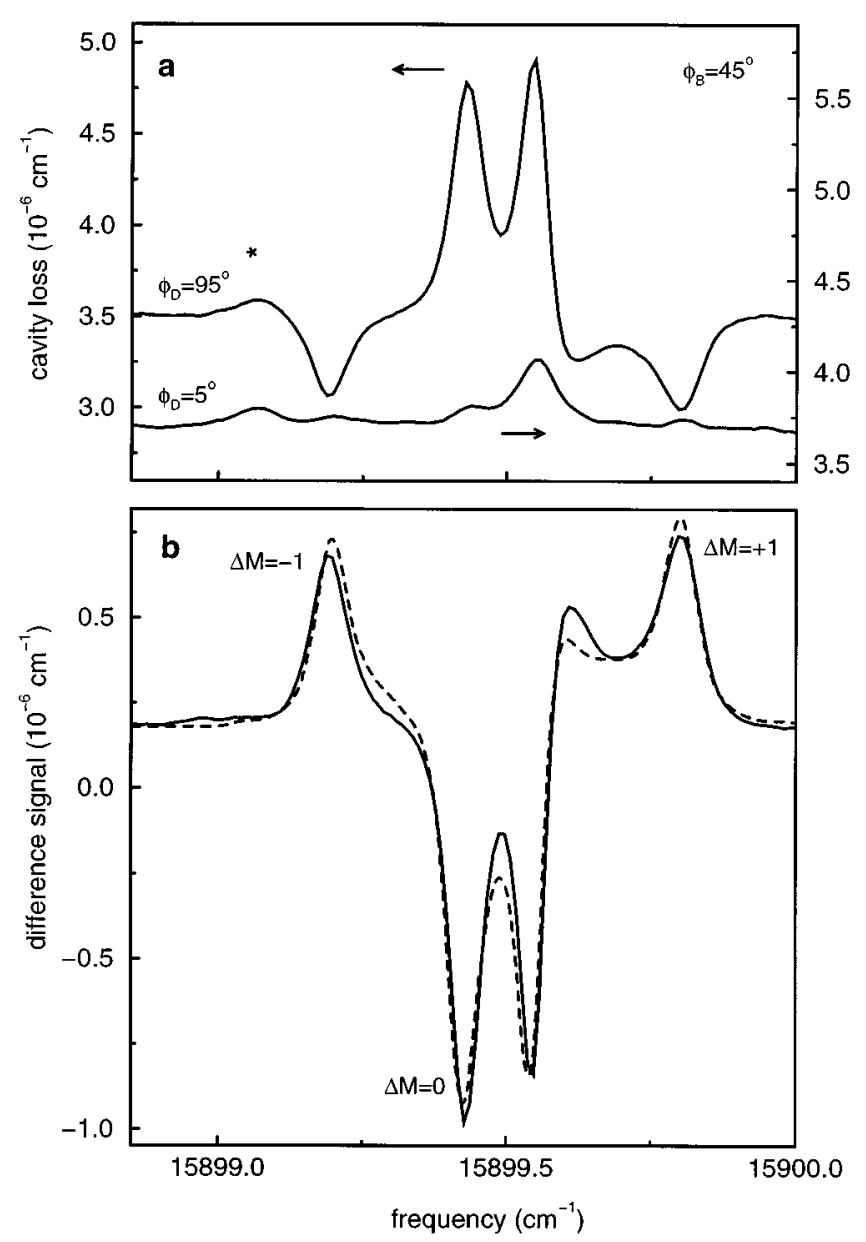

FIG. 4. The PDCRD spectrum of the ${ }^{P} P_{1}(1)$ line of the $b^{1} \Sigma_{g}^{+}\left(v^{\prime}\right.$ $=2) \leftarrow X^{3} \Sigma_{g}^{-}\left(v^{\prime \prime}=0\right)$ transition in ${ }^{16} \mathrm{O}_{2}$, measured at a pressure of $400 \mathrm{mbar}$ in the Voigt configuration, with $\phi_{B}=45^{\circ}$. The polarization dependent cavity loss spectra shown in the upper part of the figure are recorded for $\phi_{D}$ $=95^{\circ}$ and $\phi_{D}=5^{\circ}$. In the lower part of the figure the difference between the two spectra, showing exclusively the effect of polarization-dependent absorption, is shown together with the simulated spectrum according to Eq. (7). The line indicated with an asterisk is due to water absorption.

$15899.045 \mathrm{~cm}^{-1}$ is visible in the lower three spectra, with an intensity that is obviously independent of the polarization angle that is chosen.

In Fig. 4 the polarization-dependent CRD spectra of the ${ }^{P} P_{1}(1)$ transition are shown, measured with 400 mbar of $\mathrm{O}_{2}$ in the cell and with a magnetic field of $0.68 \mathrm{~T}$ perpendicular to the cavity axis. In Fig. 4(a) the cavity loss spectra as recorded for two mutually perpendicular polarization directions are shown. In this measurement the angle $\phi_{B}$ is set to $45^{\circ}$, and the polarization components that are almost perpendicular (upper trace; $\phi_{D}=95^{\circ}$ ) and almost parallel (lower trace; $\phi_{D}=5^{\circ}$ ) to the plane of polarization of the incoming beam are detected. The two spectra contain information on both the polarization independent and the polarizationdependent absorptions, as described in Eq. (6). In Fig. 4(b) the difference between the spectra for the mutually orthogonal polarization directions is shown. This difference spectrum solely shows the polarization dependent absorptions, as 
described in Eq. (7), taking $\phi_{D}=5^{\circ}$. The three individual $\Delta M$ components in the $0.68 \mathrm{~T}$ field are indicated as such in the figure. The additional structure located at a frequency close to the frequency of the field-free position is due to transitions in the fringe fields of the magnet. As the $M=0$ component shows a quadratic Zeeman shift, the $\Delta M=0$ transition in the fringe fields causes a sharp peak rather than being smeared out over a wide spectral range, as is the case for the $\Delta M= \pm 1$ components. Using the calculated Zeeman shift of the $\Delta M$ components as indicated in Fig. 2, using the measured strength of the magnetic field along the cavity axis as shown in Fig. 1, and using the known Doppler and pressure broadening of $\mathrm{O}_{2}$ in the room-temperature cell, the observed difference spectrum can be quantitatively reproduced, as shown with the dashed line in the figure. The vertical scaling of the simulation is in agreement with the absorption coefficients as extracted from Fig. 3(d) multiplied by a factor that is determined from comparing Eqs. (4) and (7). The absence of the water absorption peak from the difference spectrum explicitly demonstrates the species selectivity of the PDCRD detection scheme. It is seen in Fig. 4 that the baseline of the difference spectrum is not exactly zero, implying that there is a difference in lifetime for light polarized in mutually orthogonal directions. This difference is caused by the effective polarization rotation of the mirrors, which, although causing an offset, hardly influences the detection sensitivity in the PDCRD scheme.

The noise-equivalent absorption detection limit in the difference spectrum is found to be around $3 \times 10^{-9} \mathrm{~cm}^{-1}$, i.e., around $10^{-3}$ of the overall cavity losses. This is at least a factor of 3 better than in the CRD spectra of the individual polarization components, due to cancelation of systematic "noise." Relative to the CRD absorption spectra that are also taken with $\phi_{B}=45^{\circ}$ but without polarization selection in the detection step, as shown in Fig. 3(d), there is an additional gain in sensitivity with a factor of $2 / \sin \left(2 \phi_{D}\right)$, i.e., a factor of 11.5 for $\phi_{D}=5^{\circ}$. So apart from the species selectivity and the advantage of getting a sign-difference between absorptions polarized parallel or perpendicular to the external magnetic field, there is a demonstrated overall gain in sensitivity of more than a factor of 30 in PDCRD spectroscopy compared to "conventional" CRD absorption spectroscopy for this specific line in molecular oxygen.

\section{B. The Faraday configuration}

In Fig. 5 the PDCRD spectra of the ${ }^{P} P_{1}(1)$ transition are shown, measured with 400 mbar of $\mathrm{O}_{2}$ in the cell and with a magnetic field of $0.02 \mathrm{~T}$ parallel to the cavity axis. In Fig. 5(a) the cavity loss spectra as recorded for two mutually perpendicular polarization directions are shown. In this measurement the polarization components that are almost perpendicular (upper trace; $\phi_{D}=75^{\circ}$ ) and almost parallel (lower trace; $\left.\phi_{D}=-15^{\circ}\right)$ to the plane of polarization of the incoming beam are detected. The two spectra contain information on both the polarization (in)dependent absorptions, as well as on the optical rotation, as described in Eq. (10). In the low magnetic field employed, the individual $\Delta M$ components are

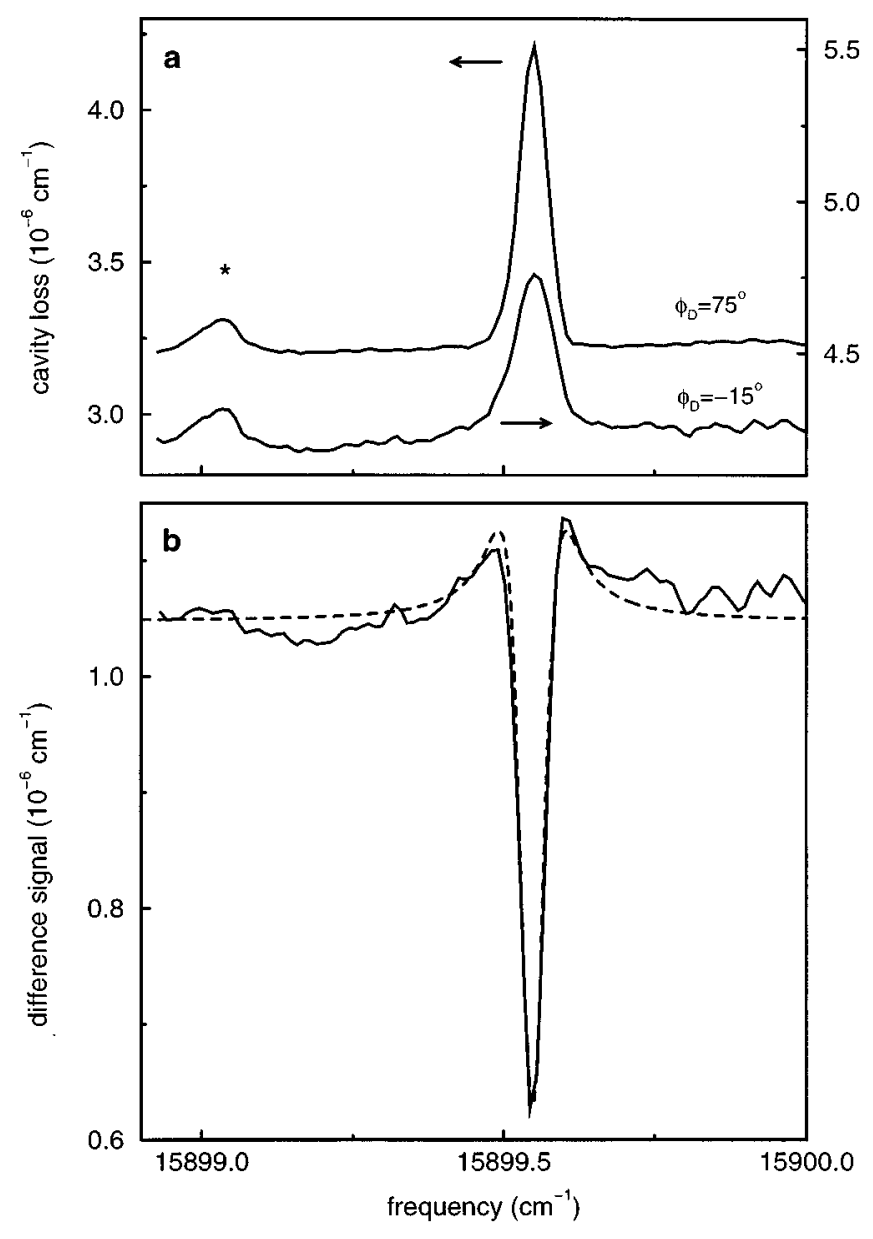

FIG. 5. The PDCRD spectrum of the ${ }^{P} P_{1}(1)$ line of the $b^{1} \Sigma_{g}^{+}\left(v^{\prime}\right.$ $=2) \leftarrow X^{3} \Sigma_{g}^{-}\left(v^{\prime \prime}=0\right)$ transition in ${ }^{16} \mathrm{O}_{2}$, measured at a pressure of $400 \mathrm{mbar}$ in the Faraday configuration. The polarization dependent cavity loss spectra shown in the upper part of the figure are recorded for $\phi_{D}=75^{\circ}$ and $\phi_{D}$ $=-15^{\circ}$. In the lower part of the figure the difference between the two spectra, showing exclusively the effect of polarization rotation, is shown together with the simulated spectrum according to Eq. (11). The line indicated with an asterisk is due to water absorption.

no longer resolved. Only the $M$-changing transitions can occur in the Faraday configuration. In Fig. 5(b) the difference between the spectra for the mutually orthogonal polarization directions is shown. This spectrum solely shows the optical rotation, as described in Eq. (11), taking $\phi_{D}=-15^{\circ}$. The observed difference spectrum can again be quantitatively reproduced in a simulation (dashed curve) in which the difference of the dispersion curves for the $\Delta M=+1$ and $\Delta M$ $=-1$ components, obtained via the Kramers-Kronig relations from the absorption curves of Fig. 3, is considered to be responsible for the observed signal. The peak intensity of the difference signal corresponds to a polarization rotation of $1.6 \times 10^{-7} \mathrm{rad} / \mathrm{cm}$. The absolute value for the difference $\left[n_{+}(\nu)-n_{-}(\nu)\right]$ in refractive indices can be determined from this spectrum. From the spectrum a noise-equivalent polarization rotation of around $10^{-8} \mathrm{rad} / \mathrm{cm}$ corresponding to an absolute polarization rotation of approximately 1.5 $\times 10^{-5}$ degree per passage through the sample is deduced in our present setup. 


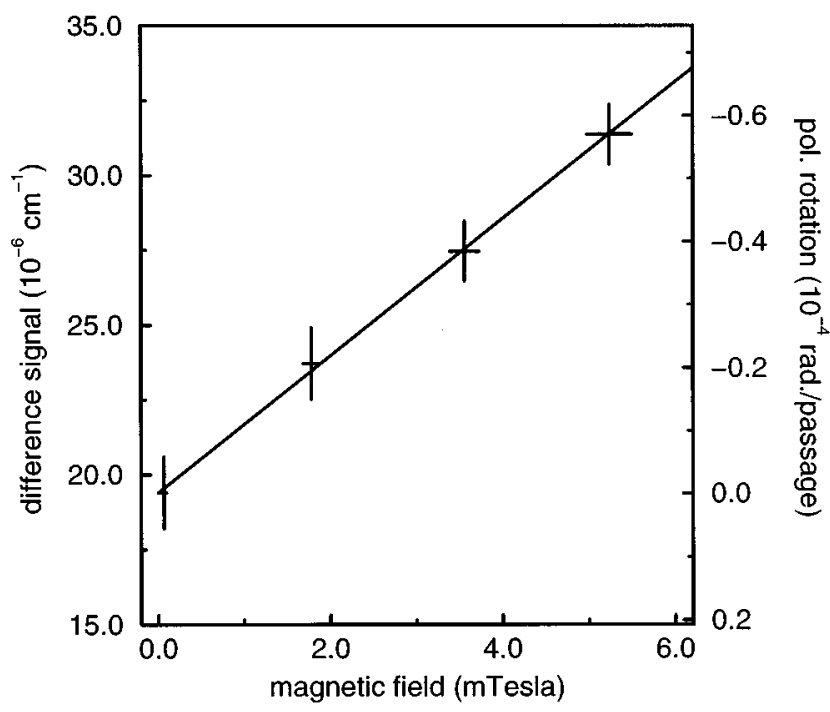

FIG. 6. The polarization rotation per passage as deduced from the PDCRD measurements on a $2 \mathrm{~mm}$ thick BK7 sample as a function of the magnetic field (Faraday configuration). The measurements are performed in vacuum at a wavelength of $618.79 \mathrm{~nm}$.

CRD absorption spectroscopy can also be applied on transparent solid samples, although to the best of our knowledge no reports of this kind have appeared in the literature yet. If an optically transparent sample is placed inside the cavity in such a way that all separate cavities are optically stable ${ }^{21}$ reflections from the surface of the sample will be captured again, and will not be noticed as overall losses from the cavity. Compared to the empty cavity there will be additional losses due to absorption in the solid as well as due to reflections of the substrate that can no longer be captured in the cavity. With a $2 \mathrm{~mm}$ thick optically flat BK7 window inserted in our ring down cavity, the overall loss per pass through the substrate could be kept below $10^{-3}$, even though the (uncoated) substrate has a reflection of about $4 \%$ per surface. These losses are still sufficiently low that sensitive absorption measurements as well as sensitive polarization rotation measurements can be performed on these samples. In order to avoid any interferences with surrounding gas (especially oxygen), the first polarizer, the ring down cavity with the sample and the coils that produce the magnetic field (see Fig. 1) are placed inside vacuum. The length of the cavity is $19.0 \mathrm{~cm}$, i.e., long enough that the ring down time can still be accurately determined, and short enough that the reflections from the surfaces of the sample stay inside the cavity. The ring down time at $\lambda=618.79 \mathrm{~nm}$, and with the 2 $\mathrm{mm}$ thick BK7 sample in the cavity and no magnetic field applied, was about $700 \mathrm{~ns}$. The light that exits the cavity after $700 \mathrm{~ns}$ passed through the sample about 1100 times. In Fig. 6 the difference in cavity loss for two mutually perpendicular polarization directions is shown as a function of the applied magnetic field, showing again an offset for zero magnetic fields. The rotation is deduced using Eq. (11) with $\phi_{D}=-15^{\circ}$. From the fit through the data points the Verdet constant of BK7 is determined to be $5.36 \mathrm{rad} \mathrm{T}^{-1} \mathrm{~m}^{-1}$ $\left(=1.84 \times 10^{4} \mathrm{~min}\right.$ of $\left.\operatorname{arc} \mathrm{T}^{-1} \mathrm{~m}^{-1}\right)$, which is in good agreement with values quoted for the Verdet constant of glasses as reported in textbooks. At magnetic fields higher than $5 \mathrm{mT}$ ring down transients that significantly deviate from a single exponentially decaying function are observed, in full agreement with Eq. (9).

\section{CONCLUSIONS}

It is theoretically outlined and experimentally verified that polarization spectroscopy can be favorably combined with cavity ring down spectroscopy. In the appropriate PDCRD detection scheme one can selectively measure either the polarization-dependent absorption or the optical rotation. In the PDCRD experiments described here, the characteristic decay times of the ring down transients for the light polarized in two mutually perpendicular directions are determined independently. As one finally is mainly interested in the difference between the polarization-dependent cavity losses, even better results are expected if the ratio of the individual ring down transients is fitted to an exponentially decaying curve. By carefully selecting the mirrors of the ring down cavity, up to two orders of magnitude can be gained in detection sensitivity in the PDCRD detection scheme relative to the "conventional" CRD absorption detection scheme. The PDCRD detection technique presented here can be implemented in all fields were cavity ring down has been used up to now. PDCRD detection will for instance enable the study of polarization-dependent kinetics. Multiplex PDCRD can be performed when a broadband excitation source is being used and when temporal and spectral analysis of the light exiting the cavity is combined. PDCRD detection can also be performed with narrow-band continuous lasers, yielding the ultimate spectral resolution. Even without temporal analysis of the light exiting the cavity, the combination of a stable optical cavity with a polarized narrow-band continuous laser can be a significant improvement over the standard multipass setup that is often employed in polarization spectroscopy. When the total, time-integrated light intensity exiting the cavity through the analyzer at an angle $\phi_{D}$ is measured, the time integral of the expressions given for the ring down transients is probed. This signal is inversely proportional to the cavity losses, and it is therefore straightforward to extract the polarization-dependent cavity losses. Using the latter approach, spectra of the ${ }^{P} P_{1}(1)$ line of molecular oxygen have been recorded with a signal-to-noise ratio that is only slightly less, but with a spectral resolution that is intrinsically much better, than that of the spectra shown in Figs. 4 and 5. Apart from studying electro-optic and magneto-optic phenomena on gas-phase species, the PDCRD detection scheme is demonstrated to be applicable to the study of optical rotation in transparent solid samples.

\section{ACKNOWLEDGMENTS}

This work is part of the research program of the "Stichting voor Fundamenteel Onderzoek der Materie (FOM)," which is financially supported by the "Nederlandse Organi- 
satie voor Wetenschappelijk Onderzoek (NWO),' and receives direct support by the NWO via PIONIER Grant No. 030-66-89.

\section{APPENDIX}

In general the electric field vector of light linearly polarized in the $\hat{x}$ direction and traveling in the $\hat{z}$ direction is represented by

$$
\mathbf{E}=E_{0} e^{i(\omega t-k z)} \hat{x} .
$$

The relation between the wave vector $k$ and the frequency $\omega$ is commonly written as $k c / \omega=n+i \kappa^{\prime}$, with $n$ and $\kappa^{\prime}$, respectively, the index of refraction at $\omega$ and the extinction coefficient at $\omega$. Using the absorption coefficient $\kappa$ $=2 \omega \kappa^{\prime} / c$, Eq. (A1) can be written as

$$
\mathbf{E}=E_{0} e^{i \omega(n z / c-t)} e^{-(1 / 2) k z} \hat{x} .
$$

\section{The Voigt configuration}

Linearly polarized light enters a ring down cavity with its plane of polarization making an angle $\phi_{B}$ with a magnetic field $\mathbf{B}$ that is oriented perpendicular to the propagation direction $\hat{z}$ of the light. The electric field components of the incoming light that are oriented parallel and perpendicular to B can be written as:

$$
\begin{aligned}
& E_{\|}=E_{0} \cos \phi_{B} e^{i \omega\left(n_{\|} z / c-t\right)} e^{-(1 / 2) \kappa_{\|} z}, \\
& E_{\perp}=E_{0} \sin \phi_{B} e^{i \omega\left(n_{\perp} z / c-t\right)} e^{-(1 / 2) \kappa_{\perp} z} .
\end{aligned}
$$

If the intensity of the light that exits the cavity is analyzed without any polarization selection, and using an absorption pathlength of $2 l$, and a roundtrip time $t=2 d / c$, one measures:

$$
I(t)=I_{0} e^{-t / \tau_{0}}\left[\cos ^{2} \phi_{B} e^{-\kappa_{\|} c l t / d}+\sin ^{2} \phi_{B} e^{-\kappa_{\perp} c l t / d}\right] .
$$

If a second polarizer is used behind the cavity, only the component of the electric field that makes an angle $\phi_{D}$ with respect to the incoming polarization $E_{\phi_{D}}$ is transmitted:

$$
E_{\phi_{D}}=E_{\|} \cos \left(\phi_{D}-\phi_{B}\right)-E_{\perp} \sin \left(\phi_{D}-\phi_{B}\right) .
$$

As the intensity measured behind the analyzer is given by $I_{\phi_{D}}(t)=\left|E_{\phi_{D}}(t)\right|^{2}$, one obtains:

$$
\begin{aligned}
I_{\phi_{D}}(t)= & I_{0} e^{-t / \tau_{0}}\left[\cos ^{2} \phi_{B} \cos ^{2}\left(\phi_{D}-\phi_{B}\right) e^{-\kappa_{\|} c l t / d}\right. \\
& +\sin ^{2} \phi_{B} \sin ^{2}\left(\phi_{D}-\phi_{B}\right) e^{-\kappa_{\perp} c l t / d} \\
& -2 \sin \phi_{B} \cos \phi_{B} \sin \left(\phi_{D}-\phi_{B}\right) \cos \left(\phi_{D}-\phi_{B}\right) \\
& \left.\times \cos \left(\frac{\omega t l}{d}\left(n_{\|}-n_{\perp}\right)\right) e^{-(1 / 2)\left(\kappa_{\|}+\kappa_{\perp}\right) c l t / d}\right] . \quad \text { (A7) }
\end{aligned}
$$

Using the approximation $1-\alpha t$ for $e^{-\alpha t}$, and writing 1 $-(1-\cos \beta t)$ for the $\cos \beta t$ term, this expression immediately leads to Eq. (5).

\section{The Faraday configuration}

A homogeneous magnetic field $\mathbf{B}$ is applied parallel to the axis of the cavity (the $\hat{z}$ axis). The linearly polarized incoming laser beam is now to be viewed as the sum of equal amounts of right- and left-circularly polarized light, respectively, $\mathbf{E}^{+}$and $\mathbf{E}^{-}$:

$$
\begin{aligned}
& \mathbf{E}^{+}=\frac{1}{2} E_{0}(\hat{x}+i \hat{y}) e^{i \omega\left(n_{+} z / c-t\right)} e^{-\kappa_{+} z / 2}, \\
& \mathbf{E}^{-}=\frac{1}{2} E_{0}(\hat{x}-i \hat{y}) e^{i \omega\left(n_{-} z / c-t\right)} e^{-\kappa_{-} z / 2} .
\end{aligned}
$$

If the light behind the cavity is measured without polarization selection, the time dependence of the light intensity is given by

$$
\begin{aligned}
I(t) & =I_{0} e^{-t / \tau_{0}} \frac{1}{2}\left[e^{-\kappa_{+} c l t / d}+e^{-\kappa_{-} c l t / d}\right] \\
& \approx I_{0} e^{-t / \tau_{0}} e^{-(1 / 2)\left(\kappa_{+}+\kappa_{-}\right) c l t / d} .
\end{aligned}
$$

If a second polarizer is used behind the cavity, only the component of the electric field that makes an angle $\phi_{D}$ with respect to the incoming polarization is transmitted:

$$
E_{\phi_{D}}=E_{x} \cos \phi_{D}+E_{y} \sin \phi_{D} .
$$

The intensity measured behind the analyzer is given by

$$
\begin{aligned}
I_{\phi_{D}}(t)= & \frac{1}{4} I_{0} e^{-t / \tau}\left(e^{-\kappa_{+} c l t / d}+e^{-\kappa_{-} c l t / d}\right. \\
& +2 \cos \left(2 \phi_{D}+\frac{\omega l t}{d}\left(n_{+}-n_{-}\right)\right) \\
& \left.\times e^{-(1 / 2)\left(\kappa_{+}+\kappa_{-}\right) c l t / d}\right),
\end{aligned}
$$

which leads to Eq. (9).

${ }^{1}$ A. O'Keefe and D. A. G. Deacon, Rev. Sci. Instrum. 59, 2544 (1988).

${ }^{2}$ A. O'Keefe, J. J. Scherer, A. L. Cooksy, R. Sheeks, J. Heath, and R. J. Saykally, Chem. Phys. Lett. 172, 214 (1990).

${ }^{3}$ J. J. Scherer, J. B. Paul, C. P. Collier, and R. J. Saykally, J. Chem. Phys. 102, 5190 (1995).

${ }^{4}$ M. G. H. Boogaarts and G. Meijer, J. Chem. Phys. 103, 5269 (1995).

${ }^{5}$ T. Yu and M. C. Lin, J. Am. Chem. Soc. 115, 4371 (1993).

${ }^{6}$ R. T. Jongma, M. G. H. Boogaarts, I. Holleman, and G. Meijer, Rev. Sci. Instrum. 66, 2821 (1995).

${ }^{7}$ J. J. Scherer, D. Voelkel, D. J. Rakestraw, J. B. Paul, C. P. Collier, R. J. Saykally, and A. O'Keefe, Chem. Phys. Lett. 245, 273 (1995).

${ }^{8}$ R. Engeln, E. van den Berg, G. Meijer, L. Lin, G. M. H. Knippels, and A. F. G. van der Meer, Chem. Phys. Lett. 269, 293 (1997).

${ }^{9}$ P. Zalicki and R. N. Zare, J. Chem. Phys. 102, 2708 (1995).

${ }^{10}$ R. Engeln and G. Meijer, Rev. Sci. Instrum. 67, 2708 (1996).

${ }^{11}$ J. Martin, B. A. Paldus, P. Zalicki, E. H. Wahl, T. G. Owana, J. S. Harris, C. H. Kruger, and R. N. Zare, Chem. Phys. Lett. 258, 63 (1996).

${ }^{12}$ K. K. Lehmann and D. Romanini, J. Chem. Phys. 105, 10263 (1996).

${ }^{13}$ J. T. Hodges, J. P. Looney, and R. D. van Zee, J. Chem. Phys. 105, 10278 (1996).

${ }^{14}$ G. Meijer, M. G. H. Boogaarts, R. T. Jongma, D. H. Parker, and A. M. Wodtke, Chem. Phys. Lett. 217, 112 (1994).

${ }^{15}$ R. Engeln, G. von Helden, G. Berden, and G. Meijer, Chem. Phys. Lett. 262, 105 (1996).

${ }^{16}$ D. Romanini, A. A. Kachanov, N. Sadeghi, and F. Stoeckel, Chem. Phys. Lett. 264, 316 (1997). 
${ }^{17}$ T. Carroll, Phys. Rev. 52, 882 (1937).

${ }^{18}$ Y. Takubo, K. Muroo, S. Miwa, K. Yamamoto, K. Suzuki, and M. Yamamoto, J. Mol. Spectrosc. 178, 31 (1996).

${ }^{19}$ H. Adams, D. Reinert, P. Kalkert, and W. Urban, Appl. Phys. B 34, 179 (1984).

${ }^{20}$ J. M. Smith, J. C. Bloch, R. W. Field, and J. I. Steinfeld, J. Opt. Soc. Am. B 12, 964 (1995).
${ }^{21} \mathrm{H}$. Kogelnik and T. Li, Proc. IEEE 54, 1312 (1966).

${ }^{22}$ H. D. Babcock and L. Herzberg, Astrophys. J. 108, 167 (1948).

${ }^{23}$ M. Tinkham and M. W. P. Strandberg, Phys. Rev. 97, 937 (1955); ibid. 97, 951 (1955).

${ }^{24}$ J. Y. Mandin, J. P. Chevillard, C. Camy-Peyret, J. M. Flaud, and J. W. Brault, J. Mol. Spectrosc. 116, 167 (1986).

${ }^{25}$ K. J. Ritter and T. D. Wilkerson, J. Mol. Spectrosc. 121, 1 (1987). 